Pacific

Journal of

Mathematics

\title{
STRONGLY SINGULAR INTEGRALS ALONG CURVES
}

Norberto LAGHI AND NeIL LyalL 


\title{
STRONGLY SINGULAR INTEGRALS ALONG CURVES
}

\author{
NORBERTO LAGHI AND NEIL LyALL
}

\begin{abstract}
We obtain $L^{2}$ bounds for strongly singular integral operators along curves in $\mathbb{R}^{d}$. Our results both generalize and extend to higher dimensions the planar results of Chandarana. In addition, we show that these operators are bounded from $L \log L$ to weak $L^{1}$ at the critical exponent $\alpha=0$.
\end{abstract}

\section{Introduction}

It is standard and well known that the Hilbert transform along curves,

$$
\mathcal{H}_{\gamma} f(x)=\text { p.v. } \int_{-1}^{1} f(x-\gamma(t)) \frac{d t}{t},
$$

is bounded on $L^{p}\left(\mathbb{R}^{d}\right)$ for $p$ in the range $1<p<\infty$, where $\gamma(t)$ is an appropriate curve in $\mathbb{R}^{d}$. This work was initiated by Fabes and Rivière [1966]. Working in $\mathbb{R}^{2}$, Nagel, Rivière, and Wainger [1974] showed that $\left\|\mathcal{H}_{\gamma} f\right\|_{p} \leq C\|f\|_{p}$, whenever

$$
\gamma(t)=\left(t, t|t|^{k}\right) \quad \text { or } \quad\left(t,|t|^{k+1}\right)
$$

with $k \geq 1$.

Stein and Wainger [1978] extended these results to well-curved $\gamma$ in $\mathbb{R}^{d}$; these $\gamma$ are smooth mappings such that $\gamma(0)=0$ and the derivatives

$$
\left.\frac{d^{k} \gamma(t)}{d t^{k}}\right|_{t=0} \text { for } k=1,2, \ldots
$$

span $\mathbb{R}^{d}$. In other words, these are smooth mappings of finite type in a small neighborhood of the origin. For the most recent results and further references, see [Christ et al. 1999].

These results notwithstanding, $\mathcal{H}_{\gamma}$ does display a certain "bad" behavior near $L^{1}$. Christ [1988] showed that $\mathcal{H}_{\gamma}$ maps the (parabolic) Hardy space $H^{1}$ into weak $L^{1}$ for the plane curves $\gamma(t)=\left(t, t^{2}\right)$. He also pointed out that $H^{1} \rightarrow$ $L^{1}$ boundedness cannot hold. A previous result of Christ and Stein [1987] had MSC2000: 44A12, 42B20.

Keywords: strongly singular integrals, Radon transforms.

Laghi was partially supported by an EPSRC grant. Lyall was partially supported by an NSF FRG grant. 
established that $\mathcal{H}_{\gamma}$ maps $L \log L\left(\mathbb{R}^{d}\right)$ into $L^{1, \infty}\left(\mathbb{R}^{d}\right)$ for a large class of curves $\gamma$ in $\mathbb{R}^{d}$.

Seeger and Tao [2001] showed that $\mathcal{H}_{\gamma}$ maps the product Hardy space $H_{\text {prod }}^{1}\left(\mathbb{R}^{2}\right)$ into the Lorentz space $L^{1,2}\left(\mathbb{R}^{2}\right)$. Their results are sharp, as $\mathcal{H}_{\gamma}$ does not map the product Hardy space into any smaller Lorentz space. Finally, Seeger, Tao, and Wright [2004] showed that $\mathcal{H}_{\gamma}$ maps $L \log \log L\left(\mathbb{R}^{2}\right)$ into $L^{1, \infty}\left(\mathbb{R}^{2}\right)$.

This short paper discusses a strongly singular analogue of the Hilbert transform along curves $\gamma(t)=\left(\gamma_{1}(t), \ldots, \gamma_{d}(t)\right)$ in $\mathbb{R}^{d}$. Namely, we consider operators of the form

$$
T_{\gamma} f(x)=\text { p.v. } \int_{-1}^{1} H_{\alpha, \beta}(t) f(x-\gamma(t)) d t,
$$

where $\beta>\alpha>0$ and $H_{\alpha, \beta}(t)=t^{-1}|t|^{-\alpha} \exp \left(i|t|^{-\beta}\right)$ is now a strongly singular (convolution) kernel in $\mathbb{R}$. This kernel enjoys some additional cancellation, as $H_{\alpha, \beta}$ is an odd function for $t \neq 0$.

Theorem 1.1. If $\gamma(t)$ is well curved, then $T_{\gamma}$ is bounded on $L^{2}\left(\mathbb{R}^{d}\right)$ if and only if $\alpha \leq \beta /(d+1)$.

Continuing the work of Zielinski [1985], Chandarana [1996] proved this theorem in $\mathbb{R}^{2}$ along the model homogeneous curves (1). Although Chandarana had some partial $L^{p}$ results, our next endpoint result near $L^{1}$ is the first for the critical value $\alpha=0$ :

Theorem 1.2. If $\gamma(t)$ is well curved, $\alpha=0$, and $\beta>0$, then $T_{\gamma}: L \log L\left(\mathbb{R}^{d}\right) \rightarrow$ $L^{1, \infty}\left(\mathbb{R}^{d}\right)$.

As a consequence of complex interpolation, one gets a result involving suitable intermediate spaces:

Corollary 1.3. If $\gamma(t)$ is well curved, then

(i) $T_{\gamma}: L^{p}(\log L)^{2(1 / p-1 / 2)}\left(\mathbb{R}^{d}\right) \rightarrow L^{p, p^{\prime}}\left(\mathbb{R}^{d}\right)$ whenever $\alpha p^{\prime} \leq 2 \beta /(d+1)$ and $1<p \leq 2$ and

(ii) $T_{\gamma}: L^{p, p^{\prime}}\left(\mathbb{R}^{d}\right) \rightarrow\left(L^{p}(\log L)^{2(1 / 2-1 / p)}\left(\mathbb{R}^{d}\right)\right)^{*}$ whenever $\alpha p \leq 2 \beta /(d+1)$ and $2 \leq p<\infty$.

Here $L^{p, q}$ denote the familiar Lorentz spaces

$$
L^{p, q}\left(\mathbb{R}^{d}\right)=\left\{f \text { measurable on } \mathbb{R}^{d}: p \int_{0}^{\infty} \lambda^{q-1}|\{x:|f(x)|>\lambda\}|^{q / p} d \lambda<\infty\right\},
$$

while the $L^{p}(\log L)^{q}$ spaces are defined by

$L^{p}(\log L)^{q}\left(\mathbb{R}^{d}\right)=\left\{f\right.$ measurable on $\left.\mathbb{R}^{d}: \int_{\mathbb{R}^{d}}|f(x)|^{p} \log ^{q}(e+|f(x)|) d x<\infty\right\}$. 
The statement of Theorem 1.2 bears an element of novelty as it is an endpoint result near $L^{1}$. As such, it is more important than the somewhat technical result of Corollary 1.3. However, while it is simple to prove that $T_{\gamma}: L^{p}\left(\mathbb{R}^{d}\right) \rightarrow L^{p}\left(\mathbb{R}^{d}\right)$ if $\alpha(d+1) / \beta<1-2|1 / p-1 / 2|$ and $1<p<\infty$, Corollary 1.3 provides a first, albeit technical, result for the conjectured sharp range of exponents $\alpha, \beta$, and $p$.

The paper is structured as follows. In the next section we shall perform some standard reductions and prove a basic oscillatory integral estimates. In Section 3, we complete the proof of Theorem 1.1. In Section 4 we give the proof of Theorem 1.2. Finally, in Section 5 we show how certain estimates found in [Seeger et al. 2004] may be applied in some special two-dimensional cases to obtain better regularity near $L^{1}$.

Notation. Throughout this paper, $C$ shall denote a strictly positive constant whose value in proofs may change from line to line and even from step to step. It depends only on the dimension $d$, on quantities such as $\alpha$ and $\beta$, and on the curve $\gamma$. Whenever we write $E=O(F)$ for any two quantities $E$ and $F$ we mean that $|E| \leq C|F|$ for some strictly positive constant $C$.

\section{2. $L^{2}$ regularity and a lemma of van der Corput type}

We first focus our attention on $L^{2}$ estimates. We shall dyadically decompose our operator $T_{\gamma}$ in the standard way. To this end, we let $\eta(t) \in C_{0}^{\infty}\left(\mathbb{R}_{+}\right)$be such that $\eta \equiv 1$ if $0 \leq t \leq 1$ and $\eta \equiv 0$ if $t \geq 2$. Then, letting $\vartheta(t)=\eta(t)-\eta(2 t)$, we have $\sum_{j \in \mathbb{Z}} \vartheta\left(2^{j} t\right) \equiv 1$ for $t>0$. We then consider the rescaled operators

$$
T_{j} f(x)=2^{j \alpha} \int \vartheta(t) t^{-1}|t|^{-\alpha} \exp \left(i 2^{j \beta}|t|^{-\beta}\right) f\left(x-\gamma\left(2^{-j} t\right)\right) d t,
$$

where, of course, $\operatorname{supp} \vartheta \subset\{t: 1 / 2 \leq|t| \leq 2\}$. Theorem 1.1 will then be a consequence of the following two results, togther with an application of Cotlar's lemma and a standard limiting argument.

Theorem 2.1 (Dyadic estimate). If $\gamma$ satisfies the finite-type condition of Theorem 1.1 , then

$$
\left\|T_{j} f\right\|_{L^{2}\left(\mathbb{R}^{d}\right)} \leq C 2^{j(\alpha-\beta /(d+1))}\|f\|_{L^{2}\left(\mathbb{R}^{d}\right)} .
$$

Proposition 2.2 (Almost orthogonality). If $\gamma$ satisfies the finite-type condition of Theorem 1.1 and $\alpha \leq \beta /(d+1)$, then the dyadic operators satisfy the estimate

$$
\left\|T_{j}^{*} T_{j^{\prime}}\right\|_{L^{2}\left(\mathbb{R}^{d}\right) \rightarrow L^{2}\left(\mathbb{R}^{d}\right)}+\left\|T_{j^{\prime}} T_{j}^{*}\right\|_{L^{2}\left(\mathbb{R}^{d}\right) \rightarrow L^{2}\left(\mathbb{R}^{d}\right)} \leq C 2^{-\delta\left|j^{\prime}-j\right|},
$$

for some $\delta>0$.

These two statements will be proved in Section 3. The key to the proofs is the following result, which is an immediate consequence (of the proof) of a lemma of Ricci and Stein [1987]; see also [Stein and Wainger 1978]. 
Lemma 2.3. Let $\varphi(t)=t^{b_{0}}+\mu_{1} t^{b_{1}}+\cdots+\mu_{n} t^{b_{n}}$ be a real-valued function, where $\mu_{1}, \ldots, \mu_{d}$ are arbitrary real parameters and $b_{0}, b_{1}, \ldots, b_{n}$ are distinct nonzero real exponents. Then

$$
\left|\int_{a}^{b} e^{i \lambda \varphi(t)} d t\right| \leq C \lambda^{-1 /(n+1)}
$$

where $C$ does not depend on $\mu_{1}, \ldots, \mu_{d}$ or $\lambda$.

Ricci and Stein in fact proved that if $b_{0}, b_{1}, \ldots, b_{n}$ are distinct positive real exponents, then

$$
\left|\int_{a}^{b} e^{i \lambda \varphi(t)} d t\right| \leq C \lambda^{\min \left\{1 / b_{0}, 1 /(n+1)\right\}}
$$

uniformly in $0 \leq a<b \leq 1$.

The analogue of (4) and (5), in which a cutoff function of bounded variation is inserted in the amplitude of the integral, follows immediately from a standard integration by parts argument.

The proof of Lemma 2.3 is essentially just that of Ricci and Stein, but we shall outline the argument here. First we recall a standard formulation of van der Corput's lemma; see [Stein 1993].

Proposition 2.4 (Van der Corput). Suppose $\psi$ is a function in $C^{k}([a, b])$ that satisfies the estimate $\left|\psi^{(k)}(x)\right| \geq C>0$ for all $x \in(a, b)$. Then

$$
\left|\int_{a}^{b} e^{i \lambda \psi(t)} d t\right| \leq k C_{k} \lambda^{-1 / k}
$$

whenever (i) $k=1$ and $\psi^{\prime \prime}(x)$ has at most one zero or (ii) $k \geq 2$.

In light of Proposition 2.4 we see that Lemma 2.3 will be a consequence of

Lemma 2.5. There exists a constant $C_{1}=C_{1}\left(b_{0}, b_{1}, \ldots, b_{n}\right)$, which is independent of $\mu_{1}, \ldots, \mu_{d}$ and $\lambda$, such that for each $t \in[a, b]$ we have $\left|\varphi^{(k)}(t)\right| \geq C_{1} t^{b_{0}-k}$ for at least one $k$ in $\{1, \ldots, n+1\}$.

To prove Lemma 2.3, we split the interval $[a, b]$ into a finite number of subintervals so that one of the inequalities of Lemma 2.5 holds on each; if the first of the inequalities holds on one, we split it further into subsubintervals on which $\varphi^{\prime}(t)$ is monotonic. The number of such subintervals depends only on $n$, and the desired conclusion follows from Proposition 2.4 and that $a$ and $b$ are contained in a compact subinterval of $(0, \infty)$.

Proof of Lemma 2.5. If we set $\mu_{0}=1$, then, for $k=1, \ldots, n+1$, we have

$$
t^{-b_{0}+k} \varphi^{(k)}(t)=\sum_{j=1}^{n+1} m_{k, j} \mu_{j-1} t^{b_{j-1}-b_{0}},
$$


where $m_{k, j}=\prod_{i=1}^{k}\left(b_{j-1}-i+1\right)$.

If we now define $w=\left(w_{1}, \ldots, w_{n+1}\right)$ with $w_{k}=t^{-b_{0}+k} \varphi^{(k)}(t)$ and define $v=\left(v_{1}, \ldots, v_{n+1}\right)$ with $v_{i}=\mu_{i-1} t^{b_{i-1}-b_{0}}$, then we have $w=M v$, where $M$ is a Vandermonde matrix with

$$
\operatorname{det} M=\prod_{j=0}^{n} b_{j} \prod_{0 \leq i<j \leq n}\left(b_{i}-b_{j}\right) .
$$

\section{The proofs of Theorem 2.1 and Proposition 2.2}

Recall that establishing $L^{2}$ estimates for the dyadic operators $T_{j}$ is equivalent to establishing uniform bounds in $\mathbb{R}^{d}$ for the multipliers

$$
m_{j}(\xi)=2^{j \alpha} \int \vartheta(t) t^{-1}|t|^{-\alpha} e^{i \psi(t)} d t,
$$

where $\psi(t)=2^{j \beta}|t|^{-\beta}-\gamma\left(2^{-j} t\right) \cdot \xi$. We shall take this multiplier approach to prove both Theorem 2.1 and Proposition 2.2.

It follows from the proposition below that we may, with no loss in generality, assume that our curves $\gamma(t)$ are of standard type, that is, they are approximately homogeneous and take the form

$$
\gamma_{k}(t)=\frac{t^{a_{k}}}{a_{k} !}+\text { higher order terms }
$$

for $k=1, \ldots, d$ with $1 \leq a_{1}<\cdots<a_{d}$.

Proposition 3.1. To every smooth well-curved $\gamma(t)$ there exists a constant nonsingular matrix $M$ such that $\tilde{\gamma}(t)=M \gamma(t)$ is of standard type.

For a simple proof of this result, see [Stein and Wainger 1978].

In the model case corresponding to the homogeneous (monomial) curves of the form $\gamma_{k}(t)=t^{a_{k}}$, we may write $\psi(t)=2^{j \beta} \varphi(t)$, where

$$
\varphi(t)=|t|^{-\beta}-\left(\mu_{1} t^{a_{1}}+\cdots+\mu_{d} t^{a_{d}}\right),
$$

with

$$
\mu=2^{-j} \circ_{\beta} \xi=\left(2^{-j\left(\beta+a_{1}\right)} \xi_{1}, \ldots, 2^{-j\left(\beta+a_{d}\right)} \xi_{d}\right) .
$$

In addition to observing that the nonisotropic dilations have entered naturally into the analysis, we also point out that Theorem 2.1 now follows immediately from Lemma 2.3 in this model case. In fact, by continuity we also obtain the estimates

$$
\left|m_{j}(\xi)\right| \leq C 2^{j(\alpha-\beta /(d+1))}
$$

for standard-type curves (7), provided the parameter $\mu$ remains bounded. 
Thus, to establish Theorem 2.1 for standard-type curves, we must obtain uniform multiplier bounds at least as strong as (8) for all large $|\mu|$. The following key result achieves more than what we need to prove Theorem 2.1 for standard type curves. The additional savings are used in a crucial way in the proof of Proposition 2.2.

Proposition 3.2 (Refined dyadic estimate). If $\gamma(t)$ is a curve of standard type, then

(i) for all $\xi \in \mathbb{R}^{d}$,

$$
\left|m_{j}(\xi)\right| \leq C 2^{j(\alpha-\beta /(d+1))}\left(1+\left|2^{-j} o_{\beta} \xi\right|\right)^{-1 /(d+1)} ;
$$

(ii) there exists a fixed $\varepsilon>0$ such that if $\left|2^{-j} o_{\beta} \xi\right| \notin\left(\varepsilon, \varepsilon^{-1}\right)$, then

$$
\left|m_{j}(\xi)\right| \leq C 2^{j(\alpha-\beta / d)}\left(1+\left|2^{-j} o_{\beta} \xi\right|\right)^{-1 / d},
$$

where $\varepsilon$ and $C$ are independent of both $j$ and $\xi$.

Proof. Modifying our approach, we write $\psi(t)= \pm 2^{j \beta} \max \left\{1,\left|2^{-j} o_{\beta} \xi\right|\right\} \varphi(t)$, with $\varphi(t)=t^{b_{0}}+\mu_{1} t^{b_{1}}+\cdots+\mu_{d} t^{b_{d}}$ and

$$
b_{0}=\left\{\begin{aligned}
-\beta & \text { if } 1 \geq \max _{k}\left\{2^{-j\left(\beta+a_{k}\right)}\left|\xi_{k}\right|\right\}, \\
\ell & \text { if } 1 \leq \max _{k}\left\{2^{-j\left(\beta+a_{k}\right)}\left|\xi_{k}\right|\right\}=2^{-j\left(\beta+a_{\ell}\right)}\left|\xi_{\ell}\right| .
\end{aligned}\right.
$$

It then follows immediately that $\left|\mu_{k}\right| \leq 1$ for all $k=1, \ldots, d$, and by continuity we obtain the estimate

$$
\left|m_{j}(\xi)\right| \leq C 2^{j(\alpha-\beta /(d+1))}\left(1+\left|2^{-j} o_{\beta} \xi\right|\right)^{-1 /(d+1)}
$$

for all curves of standard type.

It is also clear that there exists $\varepsilon>0$ such that if $\left|2^{-j} o_{\beta} \xi\right| \leq \varepsilon$, then

$$
\left|m_{j}(\xi)\right| \leq C 2^{j(\alpha-\beta)} .
$$

Now if instead we assume that $\left|2^{-j} o_{\beta} \xi\right| \geq \varepsilon^{-1}$ for some $\varepsilon>0$, then we may choose a $k$ such that

$$
2^{-j\left(b_{k}+\beta\right)}\left|\xi_{k}\right| \geq \varepsilon^{-\left(b_{k}+\beta\right)} \quad \text { and } \quad 2^{-j b_{k}}\left|\xi_{k}\right| \geq 2^{-j b_{i}}\left|\xi_{i}\right| \quad \text { for all } i \neq k .
$$

It then follows from [Ricci and Stein 1987, Lemma 2], which is the analogue of Lemma 2.5 in that setting, that if

$$
\Phi(t)=\sum_{i=1}^{d} \mu_{i} t^{b_{i}}
$$

with $\mu_{i}=2^{-j\left(b_{i}-b_{k}\right)} \xi_{i} / \xi_{k}$, then $\left|\Phi^{(\ell)}(t)\right| \geq C t^{b_{k}-\ell}$ for some $\ell$ in $\{1, \ldots, d\}$. 
It then follows from the fact that $2^{-j\left(b_{k}+\beta\right)}\left|\xi_{k}\right| \geq \varepsilon^{-\left(b_{k}+\beta\right)}$ and $\left|\mu_{i}\right| \leq 1$ for all $i=1, \ldots, d$, that

$$
\left.\left.\left|\int \vartheta(t) t^{-1}\right| t\right|^{-\alpha} e^{i\left[2^{j \beta}|t|^{-\beta}-\gamma\left(2^{-j} t\right) \cdot \xi\right]} d t\left|\leq C 2^{j b_{k} / d}\right| \xi_{k}\right|^{-1 / d}
$$

provided $\varepsilon>0$ is chosen small enough (and $j$ large enough).

Proof of Proposition 2.2. We shall only establish the desired estimate for $T_{j}^{*} T_{j^{\prime}}$. the proof of the other estimate is analogous.

It follows from Theorem 2.1 that the operators $T_{j}$ are uniformly bounded on $L^{2}\left(\mathbb{R}^{d}\right)$ whenever $\alpha \leq \beta /(d+1)$, and since we also have that $T_{j}^{*} T_{j^{\prime}} f(x)=\bar{K}_{j}(-\cdot) *$ $K_{j^{\prime}} * f(x)$, where $\widehat{K_{j}}(\xi)=m_{j}(\xi)$, we observe that

$$
\left\|T_{j}^{*} T_{j^{\prime}}\right\|=\left\|\bar{m}_{j}(\xi) m_{j^{\prime}}(\xi)\right\|_{L^{\infty}},
$$

and we can clearly assume that $\left|j^{\prime}-j\right| \gg 1$.

Let $\varepsilon>0$ be the constant given in Proposition 3.2. Without loss in generality, we assume that $j^{\prime} \geq j+C_{0}$, where $2^{C_{0}\left(\beta+a_{1}\right)} \gg \varepsilon^{-2}$. We now distinguish two cases.

(i) If $\left|2^{-j^{\prime}} o_{\beta} \xi\right| \leq \varepsilon$, it then follows from Proposition 3.2 that, for all $N^{\prime}>0$,

$$
\left|m_{j^{\prime}}(\xi)\right| \leq C 2^{j^{\prime}(\alpha-N \beta)} \leq C 2^{-j^{\prime} N^{\prime} \beta} \leq C 2^{-\left(j^{\prime}-j\right) N^{\prime} \beta} .
$$

(ii) If $\left|2^{-j^{\prime}} o_{\beta} \xi\right|>\varepsilon$, then $\left|2^{-j} o_{\beta} \xi\right| \geq C 2^{C_{0}\left(\beta+a_{1}\right)} \varepsilon \geq \varepsilon^{-1}$. Appealing to Proposition 3.2 once more, it follows that

$$
\begin{aligned}
\left|m_{j}(\xi)\right| & \leq C 2^{j(\alpha-\beta / d)}\left|2^{-j} o_{\beta} \xi\right|^{-1 / d} \\
& \leq C\left|2^{j^{\prime}-j} 2^{-j^{\prime}} \circ_{\beta} \xi\right|^{-1 / d} \\
& \leq C 2^{-\left(j^{\prime}-j\right)\left(\beta+a_{1}\right) / d}\left|2^{-j^{\prime}} o_{\beta} \xi\right|^{-1 / d} \\
& \leq C \varepsilon^{-1 / d} 2^{-\left(j^{\prime}-j\right)\left(\beta+a_{1}\right) / d} .
\end{aligned}
$$

The result then follows from estimate (10) and Theorem 2.1.

We finally comment on the necessity of the condition $\alpha \leq \beta /(d+1)$ in the statement of Theorem 1.1. It is not too difficult to see that if we consider the dyadic operator $T_{j}$ along the curve $\gamma(t)=\left(t^{a_{1}}, \ldots, t^{a_{d}}\right)$ for $t>0$ with $1 \leq a_{1}<\ldots<a_{d}$, it is possible to find constants $c_{1}, \ldots, c_{d}$ (as we shall show below) such that the multiplier $m_{j}=m_{j}(\xi)$ satisfies

$A 2^{j(\alpha-\beta /(d+1))} \leq\left|m_{j}\left(c_{1} \xi_{1}, c_{2} \xi_{1}^{\left(\beta+a_{2}\right) /\left(\beta+a_{1}\right)}, \ldots, c_{d} \xi_{1}^{\left(\beta+a_{d}\right) /\left(\beta+a_{1}\right)}\right)\right| \leq \frac{2^{j(\alpha-\beta /(d+1))}}{A}$

for some absolute constant $0<A<1$. This will imply $\left\|T_{j}\right\|_{L^{2} \rightarrow L^{2}}=\left\|m_{j}\right\|_{L^{\infty}} \approx$ $2^{j(\alpha-\beta /(d+1))}$. Observe, however, that $m_{j}(\xi)=\widehat{K_{j}}(\xi)$, where $K_{j}$ is essentially 
defined as in (11) of the next section, namely

$$
K_{j}(x)=\vartheta\left(2^{j} \rho(x)\right) K(x),
$$

where $K$ is the kernel of (2). Hence

$$
\begin{aligned}
\left|m_{j}(\xi)\right|=\left|\widehat{K}(\xi) * \vartheta \widehat{\left(2^{j} \rho(\cdot)\right)}\right| & =\left|\int \widehat{K}(\xi-\tau) \eta_{j}(\tau) d \tau\right| \\
& \leq\|\widehat{K}\|_{L^{\infty}}\left\|\vartheta \widehat{\left(2^{j} \rho(\cdot)\right)}\right\|_{L^{1}} \leq C\|\widehat{K}\|_{L^{\infty}} .
\end{aligned}
$$

In the last step, we have used that the $L^{1}$ norm of $\widehat{\vartheta\left(2^{j} \rho(\cdot)\right)}$ is normalized, which is true because this function is the Fourier transform of a rescaled bump function. Thus, $\|T\|_{L^{2} \rightarrow L^{2}} \geq C\left\|T_{j}\right\|_{L^{2} \rightarrow L^{2}}$, and our conclusion follows.

Now, to find the desired constants $c_{1}, \ldots, c_{d}$, one just has to look at which points the first $d$ derivatives of the function $\varphi=\varphi(t)$ vanish. One then obtains the system of equations

$$
\begin{array}{r}
-\beta|t|^{-\beta-1} \operatorname{sgn}(t)+2^{-j\left(\beta+a_{1}\right)} \mu_{1} a_{1} t^{a_{1}-1}+\cdots+2^{-j\left(\beta+a_{d}\right)} \mu_{d} a_{d} t^{a_{d}-1}=0, \\
\vdots \\
-\beta_{d}|t|^{-\beta-d}[\operatorname{sgn}(t)]^{d}+2^{-j\left(\beta+a_{1}\right)} \mu_{1} a_{1, d} t^{a_{1}-d}+\cdots+2^{-j\left(\beta+a_{d}\right)} \mu_{d} a_{d, d} t^{a_{d}-d}=0,
\end{array}
$$

where we have used the notation

$$
\beta_{d}=\prod_{i=1}^{d}(\beta+i-1) \quad \text { and } \quad a_{j, d}=\prod_{i=1}^{d}\left(a_{j}-i+1\right) .
$$

As this system is linear and nonsingular in the variables $\mu_{1}, \ldots, \mu_{d}$, it has a unique solution. By expressing $t$ as a function of the $\mu$ variables, one can extract the formula for the desired curve by recalling that $\mu=2^{-j} o_{\beta} \xi$ and by observing that, due to its scaling properties, no powers of $2^{-j}$ appear in the equation that gives the curve in the $\xi$ variables.

\section{Estimates near $L^{1}$}

We now turn our attention to the proof of Theorem 1.2, which relies on the result obtained by Christ and Stein [1987]. Indeed, we shall show that the general statement they proved applies to the operator (2).

We first fix some notation. For any tempered distribution $u \in \mathscr{G}^{\prime}\left(\mathbb{R}^{d}\right)$ we denote by $u^{x_{0}}$ its translate by $x_{0}$, namely,

$$
\left\langle u^{x_{0}}(x), \phi(x)\right\rangle=\left\langle u(x), \phi\left(x-x_{0}\right)\right\rangle
$$


for all test functions $\phi$. We also define the $(p, q)$ convolution norm for the operator that convolves with $u$. It is

$$
\|u\|_{C V(p, q)}=\sup _{f \in L^{p}}\|f * u\|_{L^{q}} /\|f\|_{L^{p}} .
$$

We now summarize the assumptions of the Christ-Stein theorem. Define $T$, a convolution operator, through $T f(x)=f * K(x)$, where $K$ is a tempered distribution.

Now, consider the nonisotropic dilations $x \mapsto r \circ x=\left(r^{a_{1}} x_{1}, \ldots, r^{a_{d}} x_{d}\right)$. If $\rho(x)$ is defined to be the unique $r>0$ such that $\left|r^{-1} \circ x\right|=1$, then $\rho$ becomes a quasinorm homogeneous with respect to the dilations above, see [Stein and Wainger 1978]. Thus, we may define the distributions

$$
K_{j}(x)=\vartheta\left(2^{j} \rho(x)\right) K(x) .
$$

Theorem 4.1 [Christ and Stein 1987]. Suppose $T=\sum_{j \in \mathbb{Z}} T_{j}$, where $T_{j} f=f * K_{j}$ as defined above. Assume that there exist some constants $\delta, \varepsilon>0$ such that

(i) $\left\|K_{j+\ell}-K_{j+\ell}^{x_{0}}\right\|_{C V(2,2)} \leq C 2^{-\varepsilon \ell}$ for all $y$ with $\rho(y) \leq C 2^{j}$ and all $j \in \mathbb{Z}$ and $\ell \in \mathbb{Z}_{+}$;

(ii) $\left\|K_{j}\right\|_{L^{1}} \leq C$ uniformly in $j$;

(iii) $\left\|T_{j} T_{j^{\prime}}^{*}\right\|_{L^{2} \rightarrow L^{2}}+\left\|T_{j}^{*} T_{j^{\prime}}\right\|_{L^{2} \rightarrow L^{2}} \leq C 2^{-\delta\left|j-j^{\prime}\right|}$ for all $j, j^{\prime} \in \mathbb{Z}$.

Then $T: L \log L(B) \rightarrow L^{1, \infty}(B)$ for any bounded set $B \subset \mathbb{R}^{d}$.

The above statement provides a local regularity result. However, since we are dealing with an operator given by convolution with a compactly supported kernel, one may actually use the Christ-Stein theorem to obtain a global result.

To see how the Christ-Stein theorem applies to the operator $T_{\gamma}$ in (2) when $\alpha=0$, we first consider the model case $\gamma(t)=\left(t^{a_{1}}, \ldots, t^{a_{d}}\right)$, where the $a_{j}$ are distinct positive integers. Note that the kernel $K_{\gamma}$ of $T_{\gamma}$ may be written as

$$
K_{\gamma}(x)=\iint \exp \left(i\left(|t|^{-\beta}+\xi \cdot\left(x_{1}-t^{a_{1}}, \ldots, x_{d}-t^{a_{d}}\right)\right)\right) \chi(t) t^{-1} d t d \xi .
$$

If we now define, for each $j \geq 0$,

$$
K_{\gamma, j}=\vartheta\left(2^{j} \rho(x)\right) K_{\gamma}(x)
$$

as in (11), then it is simple to see that for a test function $f$ one has

$$
\left\langle K_{\gamma, j}, f\right\rangle=\int \exp \left(i|t|^{-\beta}\right) \chi(t) t^{-1} \vartheta\left(\rho\left(2^{j} \circ\left(t^{a_{1}}, \ldots, t^{a_{d}}\right)\right)\right) f\left(t^{a_{1}}, \ldots, t^{a_{d}}\right) d t,
$$

and, as such,

$$
T_{\gamma, j} f(x)=\int \exp \left(i 2^{j \beta}|t|^{-\beta}\right) \vartheta(\rho(\gamma(t))) t^{-1} f(x-\gamma(t)) d t .
$$


It is therefore clear that the operators $T_{\gamma, j}$ are nearly identical to the operators $T_{j}$ in (3): the cutoff function found in the definition of the kernels $K_{\gamma, j}$ still restricts the $t$ variable to the set where $|t| \approx 1$. Note that trivially

$$
\left\|K_{\gamma, j}\right\|_{L^{1}} \leq C \quad \text { and } \quad\left\|T_{\gamma, j} T_{\gamma, j^{\prime}}^{*}\right\|_{L^{2} \rightarrow L^{2}}+\left\|T_{\gamma, j}^{*} T_{\gamma, j^{\prime}}\right\|_{L^{2} \rightarrow L^{2}} \leq C 2^{-\varepsilon\left|j-j^{\prime}\right|}
$$

for all $j, j^{\prime} \in \mathbb{Z}_{+}$, because the almost orthogonality of the operators $T_{\gamma, j}$ is truly equivalent to that of the operators $T_{j}$, and this has been proven in the previous section.

Thus, to apply the Christ-Stein result we need only show that

$$
\left\|K_{\gamma, j+\ell}-K_{\gamma, j+\ell}^{x_{0}}\right\|_{C V(2,2)} \leq C 2^{-\varepsilon \ell}
$$

for all $\ell \in \mathbb{Z}_{+}$and some $\varepsilon>0$. Note that $j+\ell \geq 0$, for otherwise the kernel vanishes identically. To verify this condition, it suffices to check that

$$
\left\|\widehat{K_{\gamma, j+\ell}}(\xi)-\widehat{K_{\gamma, j+\ell}^{x_{0}}}(\xi)\right\|_{L^{\infty}}=\left\|\left(1-e^{i x_{0} \cdot \xi}\right) \widehat{K_{\gamma, j+\ell}}\right\|_{L^{\infty}} \leq C 2^{-\varepsilon \ell},
$$

where

$$
\widehat{K_{\gamma, j+\ell}}(\xi)=\int \exp \left(i\left(|t|^{-\beta}+\xi \cdot\left(t^{a_{1}}, \ldots, t^{a_{d}}\right)\right)\right) \vartheta\left(2^{j+\ell} \rho(\gamma(t))\right) t^{-1} d t .
$$

First, if $j \geq 0$, there is nothing to show, as ${ }^{1}$

$$
\left|\widehat{K_{\gamma, j+\ell}}(\xi)\right| \leq C 2^{-(j+\ell) \beta /(d+1)} .
$$

However, this pointwise estimate also shows that if $j<0$, but $|j|<(1-\delta) \ell$ for some $\delta>0$, then (12) is also verified. To deal with the remaining case $j<0$ and $|j|>(1-\delta) \ell$, we note that

$$
\left|\left(1-e^{i x_{0} \cdot \xi}\right) \widehat{K_{\gamma, j+\ell}}(\xi)\right| \leq C\left|\widehat{K_{\gamma, j+\ell}}(\xi)\right| \min \left\{1,\left|\xi \cdot x_{0}\right|\right\} .
$$

Since $\left|x_{0}\right| \leq C 2^{j}$, problems may arise only if $2^{j(1-\delta)} \ll|\xi| \leq C$. Indeed, if $|\xi| \leq$ $C 2^{j(1-\delta)}$, we have the bound

$$
\left|\left(1-e^{i x_{0} \cdot \xi}\right) \widehat{K_{\gamma, j+\ell}}(\xi)\right| \leq C 2^{\delta j} \leq C 2^{-\delta \ell / 2} .
$$

Thus, consider the case $|\xi| \gg 2^{j(1-\delta)}$. Here we may use estimate (9) to get

$$
\left|\widehat{K_{\gamma, j+\ell}}(\xi)\right| \leq C 2^{-(j+\ell) \beta /(d+1)}\left(1+\left|2^{-(j+\ell)} o_{\beta} \xi\right|\right)^{-1 /(d+1)} .
$$

Using that $j<0$ and $|j|>(1-\delta) \ell$ and the size of $|\xi|$, one obtains the estimate

$$
\left(1+\left|2^{-(j+\ell)} \circ_{\beta} \xi\right|\right)^{-1 /(d+1)} \leq C 2^{-\ell / 4(d+1)},
$$

\footnotetext{
${ }^{1}$ We shall no longer explicitly mention that the kernels $K_{j}$ have the same properties as those defining the operators $T_{j}$ in (3).
} 
provided $\delta>0$ is sufficiently small. This is enough to prove (12) in this case and Theorem 1.2 for model case curves.

Passing to the general case of standard curves is not difficult. If $\gamma$ is a curve of standard type, then we again define

$$
T_{\gamma, j} f(x)=\int e^{i|t|^{-\beta}} \chi(t) t^{-1} \vartheta\left(\rho\left(2^{j} \circ \gamma(t)\right)\right) f(x-\gamma(t)) d t,
$$

where $\rho$ is homogeneous with respect to the dilations $r \circ x=\left(r^{a_{1}} x_{1}, \ldots, r^{a_{d}} x_{d}\right)$. Since $\gamma$ is approximately homogeneous with respect to the same dilations, we see that the Fourier transform of the kernel $K_{\gamma, j}$ is given by

$$
\widehat{K_{\gamma, j}}(\xi)=\int e^{i\left(2^{j \beta}|t|^{-\beta}+\xi \cdot \gamma\left(2^{-j} t\right)\right)} \vartheta\left(\rho\left(t^{a_{1}}+O\left(2^{-j}\right), \ldots, t^{a_{d}}+O\left(2^{-j}\right)\right)\right) d t .
$$

Now, for all $j>0$ sufficiently large, the cutoff function in the definition of $m_{j}$ restricts $t$ to the set where $|t| \approx 1$ and has uniformly bounded $C^{\infty}$ seminorms. Thus, the estimate of Lemma 2.3 applies, implying estimate (9), while the almost orthogonality of the operators $T_{\gamma, j}$ may be obtained as in Proposition 2.2.

To prove Corollary 1.3, one may form an analytic family of operators in the standard way and proceed as in [Chandarana 1996]. Then, the appropriate version of Stein's interpolation theorem applies. We omit the details.

\section{Estimates in two dimensions}

Seeger et al. [2004] proved a very interesting regularity result (near $L^{1}$ ) for singular Radon transforms. Let $\Sigma$ be a hypersurface in $\mathbb{R}^{d}$, and let $\mu$ be a compactly supported smooth density on $\Sigma$, that is, let $\mu=\vartheta(x) d \sigma$, where $\vartheta \in C_{0}^{\infty}\left(\mathbb{R}^{d}\right)$ and $d \sigma$ is surface measure on $\Sigma$. Let $\mu_{j}$ be dilates of $\mu$ defined by

$$
\left\langle\mu_{j}, f\right\rangle=\left\langle\mu, f\left(2^{j} \circ \cdot\right)\right\rangle,
$$

where $\circ$ denotes the nonisotropic dilations introduced in Section 4. Consider the singular Radon transform $\mathscr{R} f(x)=\sum_{j \in \mathbb{Z}} \mu_{j} * f(x)$. Assuming that the Gaussian curvature of $\Sigma$ does not vanish to infinite order at any point (in $\Sigma$ ) and that the cancellation condition $\int d \mu=0$ holds, Seeger, Tao and Wright showed that

$$
\mathscr{R}: L \log \log L\left(\mathbb{R}^{d}\right) \rightarrow L^{1, \infty}\left(\mathbb{R}^{d}\right) .
$$

It is not difficult to see that the local version $\mathscr{R}_{\mathrm{loc}} f(x)=\sum_{k<C} \mu_{k} * f(x)$ is also of weak type $L \log \log L$.

We wish to apply this result to the operator $T_{\gamma}$ in (2) in the case $d=2$ and $\alpha=0$. We also give $\gamma$ the special form $\left(t, t|t|^{b}\right)$ for $b>0$. To do so, we choose a smooth cutoff function $\vartheta=\vartheta(t)$ supported in $[1 / 2,1]$ with the property that $\sum_{j \in \mathbb{Z}_{+}} \vartheta\left(2^{j} t\right) \equiv 1$ for (say) $0<t \leq 1 / 2$. We choose another smooth cutoff $\eta$ with 
the properties that $\eta(t) \equiv 1$ for $|t| \leq M$ and $\eta(t) \equiv 0$ for $|t|>2 M$, where $M \gg 1$. Thus, if we pick the measure $\mu$ to be

$$
\mu(x)=e^{i\left|x_{1}\right|^{-\beta}} x_{1}^{-1} \vartheta\left(\left|x_{1}\right|\right) \eta\left(\left|x_{2}\right|\right),
$$

we see that its action on test function $\phi$ is given by

$$
\langle\mu, \phi\rangle=\int e^{i|t|^{-\beta}} t^{-1} \vartheta(|t|) \eta\left(t|t|^{b}\right) \phi\left(t, t|t|^{b}\right) d t=\int e^{i|t|^{-\beta}} t^{-1} \vartheta(|t|) \phi\left(t, t|t|^{b}\right) d t,
$$

provided we choose the number $M$ in the definition of $\eta$ to be large enough. Further, it is simple to see that now $\int d \mu=0$ and that the curvature of $\gamma$ does not vanish to infinite order on $[1 / 2,1]$.

Now, if we choose nonisotropic dilations $r \circ x=\left(r x_{1}, r^{b+1} x_{2}\right)$, it is simple to see that

$$
T_{\gamma} f(x)=\sum_{j \in \mathbb{Z}_{+}} \mu_{j} * f(x) .
$$

The result in [Seeger et al. 2004] then gives this:

Theorem 5.1. Let $d=2$ and $\gamma(t)=\left(t, t|t|^{b}\right)$ for $b>0$. If $\alpha=0$, then

$$
T_{\gamma}: L \log \log L\left(\mathbb{R}^{2}\right) \rightarrow L^{1, \infty}\left(\mathbb{R}^{2}\right) .
$$

If we interpolate this estimate with the sharp $L^{2}$ bounds of Theorem 1.1, we get a better regularity result (in this special two dimensional case) than the one provided by Corollary 1.3. The precise statement can be obtained by using the same procedure as in Corollary 1.3.

\section{References}

[Chandarana 1996] S. Chandarana, " $L$-bounds for hypersingular integral operators along curves", Pacific J. Math. 175:2 (1996), 389-416. MR 98b:42029 Zbl 0865.42013

[Christ 1988] M. Christ, "Weak type $(1,1)$ bounds for rough operators", Ann. of Math. (2) 128:1 (1988), 19-42. MR 89m:42013 Zbl 0666.47027

[Christ and Stein 1987] M. Christ and E. M. Stein, "A remark on singular Calderón-Zygmund theory”, Proc. Amer. Math. Soc. 99:1 (1987), 71-75. MR 88c:42030 Zbl 0622.42012

[Christ et al. 1999] M. Christ, A. Nagel, E. M. Stein, and S. Wainger, "Singular and maximal Radon transforms: analysis and geometry", Ann. of Math. (2) 150:2 (1999), 489-577. MR 2000j:42023 Zbl 0960.44001

[Fabes and Rivière 1966] E. B. Fabes and N. M. Rivière, "Singular intervals with mixed homogeneity”, Studia Math. 27 (1966), 19-38. MR 35 \#683 Zbl 0161.32403

[Nagel et al. 1974] A. Nagel, N. Rivière, and S. Wainger, "On Hilbert transforms along curves", Bull. Amer. Math. Soc. 80 (1974), 106-108. MR 56 \#9191a Zbl 0293.44002

[Ricci and Stein 1987] F. Ricci and E. M. Stein, "Harmonic analysis on nilpotent groups and singular integrals. I. Oscillatory integrals", J. Funct. Anal. 73:1 (1987), 179-194. MR 88g:42023 Zbl 0622.42010 
[Seeger and Tao 2001] A. Seeger and T. Tao, "Sharp Lorentz space estimates for rough operators", Math. Ann. 320:2 (2001), 381-415. MR 2002d:42018 Zbl 0985.42008

[Seeger et al. 2004] A. Seeger, T. Tao, and J. Wright, "Singular maximal functions and Radon transforms near $L^{1}$ ", Amer. J. Math. 126:3 (2004), 607-647. MR 2005i:44003 Zbl 1063.44003

[Stein 1993] E. M. Stein, Harmonic analysis: real-variable methods, orthogonality, and oscillatory integrals, Princeton Mathematical Series 43, Princeton University Press, Princeton, NJ, 1993. With the assistance of Timothy S. Murphy, Monographs in Harmonic Analysis, III. MR 95c:42002 Zbl 0821.42001

[Stein and Wainger 1978] E. M. Stein and S. Wainger, "Problems in harmonic analysis related to curvature", Bull. Amer. Math. Soc. 84:6 (1978), 1239-1295. MR 80k:42023 Zbl 0393.42010

[Zielinski 1985] M. Zielinski, Highly oscillatory integrals along curves, $\mathrm{PhD}$ thesis, University of Wisconsin-Madison, 1985.

Received December 20, 2006. Revised September 19, 2007.

NORBERTO LAGHI

School of Mathematics and Maxwell Institute for Mathematical Sciences

JCM BuILDING, The King's BuILDINGS

THE UNIVERSITY OF EDINBURGH

EDINBURGH EH9 3JZ

UNITED KINGDOM

N.Laghi@ed.ac.uk

NeIL LyALL

DEPARTMENT OF MATHEMATICS

Boyd Graduate Studies Research CEnTER

THE UNIVERSITY OF GEORGIA

ATHENS, GA 30602

UNITED STATES

lyall@math.uga.edu 